\title{
Aging and the Social Cognitive Determinants of Physical Activity Behavior and Behavior Change: Evidence from the Guide to Health Trial
}

\author{
Eileen Smith Anderson-Bill, ${ }^{1}$ Richard A. Winett, ${ }^{1}$ Janet R. Wojcik, $^{2}$ and David M. Williams ${ }^{3}$ \\ ${ }^{1}$ Department of Psychology (0274), Center for Research in Health Behavior, Virginia Tech Blacksburg, VA 24061, USA \\ ${ }^{2}$ Exercise Science Program, Department of Physical Education, Sport and Human Performance, 216 G Lois Rhame West Center, \\ Winthrop University, Rock Hill, SC 29733, USA \\ ${ }^{3}$ Department of Community Health, Brown University, P.O. Box G-S121-8, Providence, RI 02912, USA
}

Correspondence should be addressed to Eileen Smith Anderson-Bill, eileen@vt.edu

Received 2 September 2010; Accepted 2 February 2011

Academic Editor: Iris Reuter

Copyright ( $) 2011$ Eileen Smith Anderson-Bill et al. This is an open access article distributed under the Creative Commons Attribution License, which permits unrestricted use, distribution, and reproduction in any medium, provided the original work is properly cited.

\begin{abstract}
Part one of this study investigated the effect of aging on social-cognitive characteristics related to physical activity (PA) among adults in the baseline phase of a health promotion intervention. Participants' questionnaire responses and activity logs indicated PA levels and self-efficacy declined with age, while social support and the use of self-regulatory behaviors (e.g., goal setting, planning, and keeping track) increased. With age participants were also less likely to expect PA to interfere with their daily routines and social obligations. Part two of the study was among overweight/obese, inactive participants completing the intervention; it examined whether improvements in psychosocial variables might counteract declining PA associated with age. After treatment, participants were more active and decreased body weight regardless of age, and improved self-efficacy, outcome expectations, and self-regulatory behaviors. In a causal model, increases in self-efficacy at 7-months lead to increased PA levels and, albeit marginally, weight loss at 16 months; increased PA was associated with greater weight loss. Aging adults who were more confident exercised more and as a result lost more weight. This longitudinal study suggests interventions that offset the effect of aging on self-efficacy may be more successful in helping older participants become more active and avoid weight gain.
\end{abstract}

\section{Introduction}

The role of psychosocial functioning in adopting and maintaining healthy physical activity levels in aging adults has been highlighted by recent research [1-7]. Exercise self-efficacy, social support from significant others, positive outcome expectations, and engaging in physical activity self-regulation contribute to maintaining active lifestyles. It is important, however, to examine how aging influences psychosocial determinants of physical activity, how declines in these variables might lead to lower levels of activity, and how interventions might counteract these influences.

Social cognitive theory (SCT) provides a framework that has been recommended by the surgeon general as useful for organizing, understanding, and promoting physical activity [8]. Generally, SCT posits that personal, environmental, and behavioral factors are reciprocally influential in determining behavior and behavior change. Personal factors influencing physical activity include age, race, gender, and potentially malleable psychosocial variables, such as self-efficacy and outcome expectations. Environmental factors key to physical activity adherence involve social support, such as modeling by family and friends, support from exercise partners, and feedback from exercise leaders [9]. Behavioral factors essential to sustained physical activity are predominantly selfregulatory behaviors; Bandura suggests that, for most people exercise success depends on the ability to self-monitor (i.e., plan and track), set goals and evaluate their exercise behavior [9, page 415].

Social cognitive theory also specifies how personal, environmental, and behavioral variables relate to each other as illustrated in Figure $1[9,10]$. Self-efficacy stems from 
personal variables, such as the individual's age, gender, and general health, and from environmental variables, such as access to safe exercise facilities and social support for physical activity [9]. SCT posits that individuals who believe they can be physically active (i.e., higher self-efficacy) will expect favorable results from physical activity (i.e., outcome expectations) and will be more likely to implement the selfregulatory behaviors essential to adopting and maintaining an active lifestyle $[9,10]$.

Despite the widespread use of SCT among physical activity researchers (including studies targeting aging adults) little in known about the effect of aging on these important variables. Previous studies have generally focused on the relation between social cognitive variables and physical activity among the elderly (mean age 60-79.5) [2-5]. Very little research has examined how psychosocial variables might be influenced by advancing age or how SCT variables might mediate the influence of aging on physical activity. One exception: Ayotte et al. [1] found among older white adults married for 15 years or more (mean age $\sim 59$ years) that although the sample's physical activity levels did not decline with age, perceptions of the benefits associated with exercise did decrease.

Broader evidence will be needed to determine if deteriorating psychological functioning precedes declining physical activity levels generally associated with age [11]. Further, interventions targeting psychosocial variables to increase physical activity will need to be evaluated to determine if they operate as theorized among aging adults. The purpose of the current study was to examine the effects of age on physical activity levels and physical activity-related social support, self-efficacy, outcome expectations, and selfregulatory behavior among a diverse group of aging adults enrolled in the Guide to Health (GTH) trial [12]. Further, the study sought to examine whether the long-term effects of the SCT-based GTH intervention on physical activity levels and body weight of overweight/obese inactive aging adults were mediated by earlier changes in psychosocial variables in a manner consistent with SCT.

\section{Methods}

2.1. Recruitment and Procedures. Participants were recruited as part of a larger study to test the effectiveness of a health promotion intervention designed to reach adults living in nonmetropolitan areas through their churches [12]. In southern and rural regions of the US, regular church attendance (once a month or more) among aging adults is common (54\% of adults over age 40 in 2006) [13]. Men and women from 14 churches (3 United Methodist, 8 Baptist, and 3 predominantly African American Baptist) were recruited at their churches through meetings, newsletters, church bulletins, pulpit announcements, posters and word of mouth to participate in an Internet-based program designed to help them adopt healthier eating and physical activity habits.

Following American College of Sports Medicine guidelines [14], church members who reported heart or lung disease, asthma, diabetes, kidney/liver disease, autoimmune diseases, estimated low fitness (i.e., <3 METs) [15], bone and joint problems, or cancer within the last five years were required to have medical clearance before participating in the physical activity portion of GTH.

Research staff obtained informed consent approved by the Virginia Tech Institutional Review Board and instructed participants in how to complete paper and pencil questionnaires and the 7-Day Step Counter and Activity Log (see below); height and weight were measured during church assessment sessions. Participants returned completed questionnaires and logs to research staff at the church or via postage paid envelopes. Physical activity assessments from baseline and the 16-month followup point and psychosocial assessments at baseline and at the 7-month posttest were included in the current analyses. After baseline, participants were assigned within their churches to one of three GTH treatment conditions, GTH alone (GTH-Only), GTH plus church supports (GTH-Plus), or control waiting and were assessed immediately following the GTH intervention (7month posttest) and ten months later (16-month followup).

Baseline participants over 40 years of age $(n=703)$ contributed data to part one of the current study. Of baseline participants over 40,73\% $(n=515)$ completed the 16 month followup assessment; 204 were classified as "weight challenged and inactive" (i.e., BMI $\geq 25$; daily steps $<7500$ [16]; moderate intensity exercise $<30$ minutes 5 days a week and vigorous intensity exercise $<20$ minutes 3 days a week [17]). Weight challenged and inactive adults over 40 who completed the 16-month assessment contributed data to part two of the current study.

\subsection{The Guide to Health Intervention. The Internet-based} GTH program, accessible from any computer connected to the Internet, consisted of 12 weekly SCT-based modules of 15-20 screens [12]. Modules targeted social support (e.g., getting someone to remind you to walk; adding healthier foods in ways acceptable to family members), self-efficacy (i.e., guided, gradual behavior change, e.g., increasing steps 500 steps per/day and increasing $\mathrm{F} \& \mathrm{~V}$ by one serving per day), outcome expectations, (i.e., providing feasible and acceptable strategies, e.g., building steps into one's normal routine and switching to acceptable fat- modified foods), and self-regulation (i.e., anticipating and planning for barriers to change, e.g., walking at the mall in bad weather, bringing fruit for a snack when healthy alternatives are not available at the workplace).

Church-based supports provided in churches in one study condition included prompts from the pulpit and in church bulletins, reports of church progress toward behavior change goals, and a church-wide "step-drive" [12] and were designed to garner support for behavior change from the churches' social networks. Church supports were faded after the 7-month assessment and ended prior to the 16-month assessment.

\section{Measures}

\subsection{Physical Activity}

3.1.1. Verified Step-Counts. Participants received a pedometer (Accusplit 120E step counter; San Jose, CA) and a "7-Day 
TABle 1: Physical activity beliefs survey: scale descriptions.

\begin{tabular}{|c|c|c|c|}
\hline Variable description & Sub-scale & \# items & $\alpha$ \\
\hline Social support from family for physical activity & & 3 & .68 \\
\hline \multirow{2}{*}{ Self Efficacy } & Overcoming barriers & 11 & .89 \\
\hline & Meeting goals in daily routine & 9 & .89 \\
\hline Positive physical, and self-evaluative expectations & & 3 & .81 \\
\hline Negative physical, social, and self-evaluative expectations & & 6 & .85 \\
\hline Self regulation (se of goal setting, planning, and self-monitoring) & & 7 & .83 \\
\hline
\end{tabular}

$\alpha=$ Cronbach's alpha coefficient of internal consistency.

Step Counter and Physical Activity Log" to keep track of their physical activity for one week. Participants wore the pedometer and made a daily record of steps accumulated during the week. Participants were instructed to not reset their pedometers during the week and to let the steps accumulate until the seventh day. Step-logs and pedometers were returned to the research site where staff used the accumulated step-count reading on the pedometer to verify steps logged for the week [18]. Mean daily step counts (total steps $\div$ days of pedometer use) served as one measure of physical activity in the baseline model in part one of the study. Change in mean daily step counts (16-month assessment minus baseline) served as one measure of physical activity in the behavior change model in part two of the study.

3.1.2. Physical Activity Log. In addition to logging steps, participants were asked to record each morning, afternoon and evening for one week "any physical activity comparable to how you feel when you are walking at a normal walking pace." For each participant, the number of minutes spent walking per day was computed. Minutes walked per day served as a second measure of physical activity in the baseline model in part one. In addition, the total number of MET minutes engaged in exercise was summed across logged activities to calculate exercise MET-hours/week. Exercise was defined as planned, structured, and repetitive body movement done to improve or maintain ...physical fitness [14]. The MET equivalent (i.e., the ratio of work metabolic rate to a standard resting metabolic) for each logged activity was computed. Based on Ainsworth [19] activities of moderate or higher intensity ( $\geq 3 \mathrm{METs}$ ) done to maintain/improve fitness that lasted at least 10 minutes, were used to compute exercise MET-hours/week. Change in participants' exercise MET-hours/week (16-month assessment minus baseline) served as a second measure of physical activity in the behavior change model in part two. (Note: the nonlinear relationship between age and MET-hours/week at baseline prevented its use in the baseline model of part one).

3.2. Social Cognitive Variables. The Physical Activity Beliefs section of the Health Beliefs Survey [18] measured physical activity-related social support, self-efficacy, outcome expectations and self-regulation (see Table 1). Baseline scores for social cognitive variables were used as measures in the baseline model. Change scores (baseline scores subtracted from scores at 7 months) were used as measures of SCT variables in the behavior change model.

\section{Statistical Methods}

Structural models were analyzed with latent-variable structural equation modeling (SEM, LISREL 8.8, [20]); model fit was evaluated with root mean square error of the approximation $(R M S E A) \leq .05$ ( $P$ close fit $>.95$ or alpha $=.05)$ and with Chi-square evaluated with alpha $=.05$ or $<3$ times degrees of freedom (normed chi-square; [21]). We assumed no measure to be error free, so, for latent variables with only one indicator (e.g., age, gender, and race), we set error terms to the measure's variance times estimated error [21]. In order to make full use of the available data, full information maximum likelihood estimation was employed. Prior to analyses, measures were examined for outliers and normality. The distributions of the baseline physical activity measures and, with few exceptions, the distributions of change measures were skewed or displayed unacceptable kurtosis; these measures were normalized using the Blom proportional estimate formula in SPSS 18.0.

Using procedures appropriate for evaluating change in group-randomized trials [22], complex-sample, latentvariable SEM with 14 clusters (churches) nested in 3 ordinal study conditions $(0=$ control, $1=$ GTH, $2=$ GTH Plus Supports) evaluated whether the effects associated with level of GTH treatment on physical activity were mediated by underlying SCT variables. Further, the behavior change model was re-evaluated to determine if and how change in physical activity (and preceding changes in social cognitive variables) were related to differences in weight at 16 months.

In both parts of the study, effect mediation (e.g., mediation of the effect of age on physical activity or the effect of treatment on change in physical activity) was examined when (1) the predictor variable had a significant total effect on the outcome variable, (2) the predictor variable had a significant total effect on the presumed mediating variable, and (3) the mediating variable had a significant total effect on the outcome variable (evaluated one-tailed in the direction of the hypothesized effect [23-26]). Mediation was evaluated with $z^{\prime}$ [25], which in its various versions consistently had more power and lower Type 1 error than other mediation tests [25$27]$ including in cluster randomized designs $[22,28,29] . Z^{\prime}$ was used to determine upper and lower critical values for the mediated effect $(a b)$ based on an empirical distribution 
of indirect effects [25]; lower and upper confidence limits of $a b$ that did not include zero were interpreted as a significant mediating effect.

\section{Results}

\subsection{Part One: Demographic, Psychosocial, and Behavioral Characteristics.}

5.1.1. Demographic Characteristics. Participants in part one of the study ( $n=703$ ) had a mean age of 58.11 years $(\mathrm{SD}=11.08), 23 \%$ were African American, $66 \%$ female, $90 \%$ attended church at least once a week, 20\% reported annual household incomes less than $\$ 20,000$ (median income $\sim \$ 50 \mathrm{k}) ; 22 \%$ reported 12 years or less education $(M=14.8$, $\mathrm{SD}=2.4), 75 \%$ were overweight or obese, and $48 \%$ were inactive. About half $(n=339)$ of the over-40 participants reported one or more health problems requiring clearance to participate in the PA intervention; 99\% received written medical clearance. The number of participants in each health issue category was as follows: 220 reported heart disease, 133 bone or joint problems, 101 pulmonary disease, 82 diabetes, 73 thyroid disease, and 39 other diseases.

5.1.2. Physical Activity Levels. Baseline step-count and activity logs indicated the over-40 participants took an average of 6507.20 steps $(S D=3252.52)$ and reported walking an average of 23.95 minutes $(S D=32.64)$ per day during the initial assessment phase. A comparison of physical activity among 40s-group ages 40-51 (the 40s-group, $n=213$ ), ages 52-61 (the 50s-group; $n=241$ ), age 62 and up (the 60s+-group, $n=249$ ) revealed that activity levels decreased significantly with age. Participants in the 40 s-group took $26 \%$ more steps and walked $40 \%$ more minutes per day than participants in the 60s+-group (see Table 2 ).

5.1.3. Perceived Social Support for Physical Activity. Responses to the Physical Activity Beliefs Survey indicated that, prior to intervention, participants' perceived some, although not strong, social support from their families for physical activity $(M=3.44, \mathrm{SD}=.85$ on a $1-5$ scale $)$. Perceived social support was highest among participants in the 60s+-group compared to participants in the 40s- and 50 s-group, and higher among the 50s-group than participants the 40s-group (see Table 2).

5.1.4. Self-Efficacy. Mean self-efficacy scores indicated participants had positive, but not complete, confidence in their abilities to increase physical activity in their daily lives $(M=$ $72.54, \mathrm{SD}=20.04$ on a 100 point scale). Participants' confidence in being able to overcome barriers to physical activity, on the other hand, was more neutral $(M=58.17$, $\mathrm{SD}=21.62100$ point scale). Unlike social support which increased with age, participants' self-efficacy for physical activity decreased. Participants in the 60s+-group reported lower confidence in their abilities to add exercise to their daily routines than participants in the 40s- and 50s-groups, and self-efficacy for overcoming barriers to exercise was higher in the 40s-group than the 60s+-group (see Table 2).

5.1.5. Outcome Expectations. Responses to the negative outcome expectations items indicated participants did not expect that increasing physical activity would interfere with social and time management responsibilities $(M=7.66$, $\mathrm{SD}=4.05$ on a 25 point valued outcome 5 expectations scale). Negative outcome expectations declined (improved) with age; participants in the 60s+-group had lower negative outcome expectations than those in the 40s-group (see Table 1). Participants did, however, expect positive outcome from being more active-agreeing that increasing physical activity would lead them to better physical and emotional health $(M=18.56$; SD $=5.58$ on a 25 point valued expectations scale). Although, the 50s-group had higher positive outcome expectations than the 60s+-group (see Table 2), these groups did not differ from the 40s-groups.

5.1.6. Self-Regulation. Finally, participants indicated they seldom (rated 2 on the 5 point scale) or occasionally ( 3 on the scale) implemented physical activity self-regulatory behaviors in the three months prior to the intervention $(M=2.41, \mathrm{SD}=.89)$. These behaviors, however, increased with age and were more frequent among participants in the 60 s+-group as compared to participants in the 40s-group (see Table 2).

5.2. Part One: Social Cognitive Determinants of Physical Activity among Aging Adults. The nature of the relationships between baseline social cognitive variables, physical activity, age, gender, race, and health status was investigated by modeling these variables in a manner consistent with SCT and evaluating the model with structural equation analysis. The SCT model of baseline physical activity (see Figure 2) provided a good fit to the data $(R M S E A=.046, P$ (close fit) $=.97 ; X_{(392, N=703)}^{2}=975.14, P<.001 ; X^{2} /$ df ratio $\left.=2.49\right)$ explaining $18 \%$ of the variance in physical activity observed among the aging adults. Standardized direct, indirect, and total effect coefficients generated by the structural analysis are listed in Table 3; significant direct effects are printed in Figure 2 (covariance matrices and factor loadings associated with the analyses are available from Eileen Anderson-Bill).

5.2.1. Age, Gender, Race, and Health Status. Within the model, age exerted the strongest total effect on physical activity $\left(\beta_{\text {(total })}=-.41\right.$; see the last row in Table 3$)$; greater age was associated with lower levels of physical activity. Age also influenced social support $\left(\left(\beta_{\text {(total })}=.37\right)\right.$, self-efficacy $\left(\beta_{\text {(total })}\right.$ $=-.14)$, negative outcome expectations $\left(\beta_{(\text {total })}=-.23\right)$, and self-regulation $\left(\beta_{\text {(total })}=.25\right)$.

Participants' race also influenced physical activity $\left(\beta_{\text {(total })}\right.$ $=-.24)$; African American participants had lower levels of physical activity than participants of other races $(97 \%$ of whom were white). African American older adults also expected more positive outcomes $\left(\beta_{(\text {total })}=.09\right)$ and fewer negative outcomes $\left(\beta_{\text {(total })}=-.12\right)$ than white participants. 
TABLE 2: Age-related differences in physical activity and related social cognitive variables.

\begin{tabular}{|c|c|c|c|c|c|c|c|}
\hline & Age group & Mean & SD & $F$ & $P$ & Compared Groups & $t(p)$ \\
\hline \multirow{3}{*}{ Steps/day } & $40 \mathrm{~s}$ & 7315.67 & 3054.50 & 23.95 & $<.001$ & 40 s versus 50 s & $-1.34(.54)$ \\
\hline & $50 \mathrm{~s}$ & 6918.41 & 3363.12 & & & 40 s versus 60 s + & $6.45(<.001)$ \\
\hline & $60 s+$ & 5417.64 & 3017.65 & & & 50 s versus $60 \mathrm{~s}+$ & $5.27(<.001)$ \\
\hline \multirow{3}{*}{ Min walked/day } & $40 \mathrm{~s}$ & 27.93 & 38.83 & 3.43 & .03 & 40 s versus 50 s & $.93(.44)$ \\
\hline & $50 \mathrm{~s}$ & 24.93 & 31.34 & & & 40 s versus 60 s+ & $2.56(.04)$ \\
\hline & $60 s+$ & 19.83 & 27.61 & & & 50 s versus $60 \mathrm{~s}+$ & $1.66(.96)$ \\
\hline \multirow{3}{*}{ Social support } & $40 \mathrm{~s}$ & 3.17 & .79 & 18.41 & $<.001$ & 40 s versus $50 \mathrm{~s}$ & $-3.67(<.001)$ \\
\hline & $50 \mathrm{~s}$ & 3.48 & .83 & & & 40 s versus 60 s + & $-6.04(<.001)$ \\
\hline & $60 s+$ & 3.66 & .87 & & & 50 s versus 60 s + & $-2.46(.04)$ \\
\hline \multirow{3}{*}{ SE: daily routine } & $40 \mathrm{~s}$ & 74.38 & 17.78 & 4.25 & .02 & 40 s versus $50 \mathrm{~s}$ & $-.55(1.00)$ \\
\hline & $50 \mathrm{~s}$ & 75.43 & 18.65 & & & 40 s versus $60 \mathrm{~s}+$ & $3.33(<.001)$ \\
\hline & $60 s+$ & 68.04 & 22.41 & & & 50 s versus $60 \mathrm{~s}+$ & $4.01(<.001)$ \\
\hline \multirow{3}{*}{ SE: barriers } & $40 \mathrm{~s}$ & 58.13 & 19.97 & 9.31 & $<.001$ & 40 s versus $50 \mathrm{~s}$ & $-1.44(.45)$ \\
\hline & $50 \mathrm{~s}$ & 61.13 & 20.81 & & & 40 s versus $60 \mathrm{~s}+$ & $1.39(.50)$ \\
\hline & $60 s+$ & 55.26 & 23.45 & & & 50 s versus $60 \mathrm{~s}+$ & $2.92(.01)$ \\
\hline \multirow{3}{*}{ Negative OE } & $40 \mathrm{~s}$ & 8.07 & 3.94 & 3.71 & .03 & 40 s versus 50 s & $.51(1.00)$ \\
\hline & $50 \mathrm{~s}$ & 7.87 & 4.22 & & & 40 s versus 60 s + & $2.55(.03)$ \\
\hline & $60 s+$ & 7.03 & 3.90 & & & 50 s versus $60 \mathrm{~s}+$ & $2.11(.11)$ \\
\hline \multirow{3}{*}{ Positive OE } & $40 \mathrm{~s}$ & 18.76 & 5.41 & 3.59 & .03 & 40 s versus 50 s & $-.73(1.00)$ \\
\hline & $50 \mathrm{~s}$ & 19.16 & 5.45 & & & 40 s versus 60 s+ & $1.83(.21)$ \\
\hline & $60 s+$ & 17.73 & 5.80 & & & 50 s versus $60 \mathrm{~s}+$ & $2.62(.03)$ \\
\hline \multirow{3}{*}{ SR: goal setting and planning } & $40 \mathrm{~s}$ & 2.50 & .88 & 5.68 & $<.001$ & 40 s versus $50 \mathrm{~s}$ & $-1.55(.36)$ \\
\hline & $50 \mathrm{~s}$ & 2.64 & .96 & & & 40 s versus 60 s + & $-3.36(<.001)$ \\
\hline & $60 s+$ & 2.80 & .95 & & & 50 s versus 60 s + & $-1.85(.20)$ \\
\hline \multirow{3}{*}{ SR: tracking } & $40 \mathrm{~s}$ & 1.73 & 1.01 & 4.18 & .02 & 40 s versus 50 s & $-1.24(.65)$ \\
\hline & $50 \mathrm{~s}$ & 1.87 & 1.15 & & & 40 s versus 60 s + & $-2.87(.01)$ \\
\hline & $60 s+$ & 2.05 & 1.23 & & & 50 s versus $60 \mathrm{~s}+$ & $-1.68(.28)$ \\
\hline
\end{tabular}

SE: self-efficacy, OE: outcome expectations, SR: self-regulation.

Social support, self-efficacy, and self-regulation among aging adults were not influenced by race.

Gender (female $=0$, male $=1$ ) did not exert an overall effect on physical activity (i.e., its total effect was insignificant). Women in the sample, however, had higher levels of self-efficacy $\left(\beta_{\text {(total })}=-.09\right)$, positive outcome expectations $\left(\beta_{(\text {total })}=-.19\right)$, and self-regulatory behavior $\left(\beta_{(\text {total })}=-.14\right)$.

Participants who reported health concerns requiring medical clearance (see above) were less physically active than participants who did not $\left(\beta_{\text {(total })}=-.14\right)$, had lower levels of physical activity self-efficacy $\left(\beta_{\text {(total })}=-.09\right)$, and higher negative outcome expectations $\left(\beta_{(\text {total })}=.12\right)$ for physical activity, but the effect of health status on physical activity was larger independent of these variables $\left(\beta_{\text {(direct })}=-.14\right)$.

5.2.2. Social Support. Social support from family members contributed to aging adults' physical activity levels ( $\beta_{\text {[total] }}$ $=.12, P<.05)$; an effect that was largely indirect through self-efficacy and self-regulation $(\beta$ [indirect] $=.10, P<$ .001 ; indirect/total ratio $=.83)$. Social support was strongly predictive of self-efficacy $\left(\beta_{\text {[total }]}=.34, P<.001\right)$ and of whether aging adults engaged in self-regulatory behavior $\left(\beta_{\text {[total }]}=.43, P<.001\right)$. Participants who perceived support from their families for physical activity were more likely to expressed confidence in their abilities to fit exercise into their daily routines and to overcome barriers to physical activity; they were also more likely to set goals, plan, and self-monitor their own activity levels. The effect of social support on selfregulation was largely direct $(\beta$ [indirect] $=.06, P<.05$; indirect/total ratio $=.12$ ).

5.2.3. Self-Efficacy. Although self-efficacy decreased with age in the sample, aging adults with greater confidence in their being able to manage the logistics and to overcome barriers to physical activity were more active; this moderate effect was almost entirely direct $\left(\beta_{\text {[total }]}=.12, P<.05 ; \beta\right.$ [indirect] $=-.02, P>.10$; indirect/total ratio $=.16$ ). In addition to influencing physical activity, self-efficacy was a strong predictor of outcome expectations in the model (negative outcome expectations; $\beta_{\text {[total }]}=-.27, P<.001$; positive outcome expectations; $\left.\beta_{\text {[total }]}=.41, P<.001\right)$ and had a moderate effect on self-regulation $\left(\beta_{\text {[total] }}=.17, P<.001\right)$. Participants with confidence in their abilities to maintain an active lifestyle were more likely to expect to reap the benefits from becoming more active and were more likely to engage in self-regulatory behavior. 


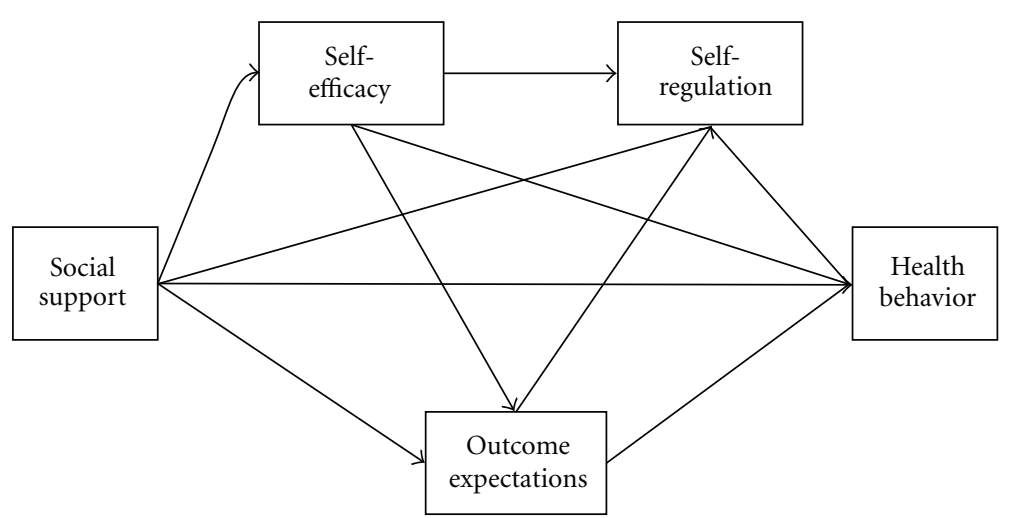

FIGURE 1: Variable relationships within the social cognitive model of health behavior.

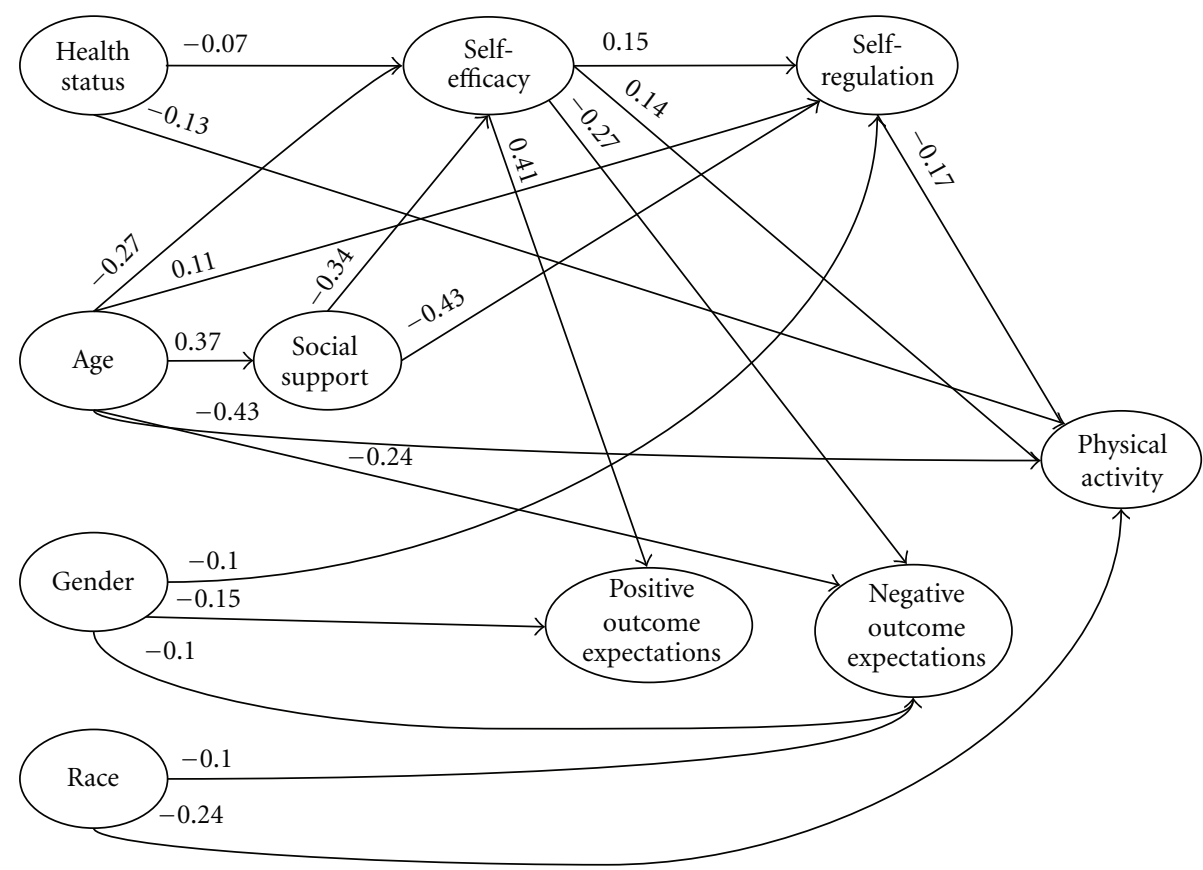

FIGURE 2: Social cognitive model of physical activity among aging adults: significant direct effects $(P<.05)$.

5.2.4. Outcome Expectations. Outcome expectations did not exert a significant effect on aging adults' physical activity (negative outcome expectations; $\beta_{\text {[total }]}=.06, P<.10$; positive outcome expectations; $\beta_{\text {[total }]}=-.07, P>.10$ ) nor did outcome expectations influence participants' use of selfregulatory behaviors (negative outcome expectations; $\beta$ [total] $=-.01, P<.10$; positive outcome expectations; $\beta_{[\text {total }]}=.05$, $P>10)$.

5.2.5. Self-Regulation. Enactment of self-regulatory behaviors was a moderate predictor of aging adults' physical activity. Setting activity goals and making plans, adjusting routines to make activity more enjoyable, and tracking daily activity led to higher levels of walking $\left(\beta_{[\text {total }]}=.17, P<\right.$ $.001)$. Indeed, self-regulation mediated the effect of age in the sample (Age-Self-regulation-Physical Activity $a b=.055$; $\mathrm{CI}=.01 ; .12)$.
5.2.6. Potential SCT Mediators of Age and Health Status Effects on Activity Levels. Age and health status were important predictors of physical activity and related psychosocial variables. The extent to which the effects of age and health status were mediated by SCT variables was further investigated. The effects of age on physical activity were mediated by social support, self-efficacy, and self-regulation. Older participants' decreased self-efficacy contributed to lower levels of physical activity (Age-Self-Efficacy-Physical Activity $a b=-.02$; CI $=-.04 ;-.001)$. On the other hand, the increased socialsupport (Age-Social Support-Physical Activity $a b=.04$; $\mathrm{CI}=.002 ; .09$ ) and self-regulation (Age-Self-regulationPhysical Activity $a b=.04 ; \mathrm{CI}=.004 ; .09)$ associated with greater age contributed to higher levels of physical activity acting as a counterbalance to the negative effects of declining self-efficacy resulting in an insignificant total indirect effect of age on physical activity $(\beta$ [indirect] $=.02, P=.30)$. 
TABLE 3: Standardized direct, indirect, and total effects among latent variables from the social cognitive model of baseline physical activity among aging adults.

\begin{tabular}{|c|c|c|c|c|c|c|c|c|c|c|}
\hline Latent variable & & Race & Gender & Age & Health & SS & SE & POE & NOE & SR \\
\hline Social support (SS) & Direct/total & .02 & -.05 & $.37^{* * *}$ & -.05 & & & & & \\
\hline \multirow{3}{*}{ Self-efficacy (SE) } & Direct & .05 & -.07 & $-.27 * * *$ & -.07 & $.34 * * *$ & & & & \\
\hline & Indirect & .01 & -.02 & .13 & -.02 & - & & & & \\
\hline & Total & .06 & $-.09^{*}$ & $-.14^{* *}$ & $-.09^{*}$ & $.34^{* * *}$ & & & & \\
\hline \multirow{3}{*}{ Positive outcome expectations (POE) } & Direct & .06 & $-.15^{* *}$ & -.04 & .01 & .05 & $.41^{* * *}$ & & & \\
\hline & Indirect & .02 & -.04 & -.04 & -.04 & $.14^{* * *}$ & - & & & \\
\hline & Total & $.09 *$ & $-.19^{* * *}$ & -.08 & -.03 & $.19^{* *}$ & $.41^{* * *}$ & & & \\
\hline \multirow{3}{*}{ Negative outcome expectations (NOE) } & Direct & -.10 & $-.10^{*}$ & $-.24^{* * *}$ & .09 & -.08 & $-.27 * * *$ & & & \\
\hline & Indirect & -.02 & .03 & .01 & .03 & $-.09^{* *}$ & - & & & \\
\hline & Total & $-.12^{* *}$ & -.07 & $-.23 * * *$ & $.12^{* *}$ & $-.17^{* * *}$ & $-.27 * * *$ & & & \\
\hline \multirow{3}{*}{ Self-regulation (SR) } & Direct & .06 & $-.10^{*}$ & .11 & .00 & $.43^{* * *}$ & $.15^{* *}$ & .05 & -.01 & \\
\hline & Indirect & .02 & -.04 & .14 & -.04 & $.06^{* *}$ & .02 & - & - & \\
\hline & Total & $.09^{\mathrm{a}}$ & $-.14^{* *}$ & $.25^{* * *}$ & -.04 & $.49^{* * *}$ & $.17^{* * *}$ & .05 & -.01 & \\
\hline \multirow{3}{*}{ Physical activity } & Direct & $-.24^{* *}$ & .02 & $-.43^{* * *}$ & $-.13^{* *}$ & .01 & $.14^{*}$ & -.08 & .06 & $.17^{* *}$ \\
\hline & Indirect & .01 & -.03 & .02 & -.01 & $.10^{* * *}$ & -.02 & .01 & .00 & - \\
\hline & Total & $-.24^{* * *}$ & -.01 & $-.41^{* * *}$ & $-.14^{* *}$ & $.12^{*}$ & $.12^{*}$ & -.07 & .06 & $.17^{* *}$ \\
\hline
\end{tabular}

a $P<.10 ; * P<.05 ; * * P<.01 ; * * * P<.001$.

Increased social support associated with aging also tempered the negative of age on self-efficacy (Age-Social-SupportSelf-Efficacy $a b=.13$; CI $.07 ; .18$ ) illustrated by comparing total and direct effects of aging on self-efficacy in Table 3. Similarly, poor health among aging participants made it more likely they had lower self-efficacy for exercise which mediated the effect of health status on physical activity (Health Status- Self-Efficacy-Physical Activity $a b=-.01$; $\mathrm{CI}=-.03 ;-.00004)$.

5.3. Part Two: Social Cognitive Mediators of Guide to Health Effects among Aging Adults. The extent to which GTHrelated changes in psychosocial variables might counteract declining physical activity and increased weight associated with age was investigated among inactive weight-challenged aging adults completing the GTH trial with a complexsample, longitudinal, latent variable approach to SEM. The models followed SCT incorporating change data for SCT variables computed from the baseline and seven-month assessments (i.e., change variable $=7$ months variable minus baseline variable) and incorporating change data for physical activity and weight variables from the baseline and 16-month assessments (i.e., change variable $=16$-month variable minus baseline variable). Means and standard deviations of measured variables in the latent-variable model, reported by study condition, are displayed in Table 4.

5.3.1. Guide to Health Effects on Physical Activity. The SCT model of treatment effects on physical activity (see Figure 3) provided good fit to the data: $R M S E A=.00$, $95 \%$ CI $=.00 ; .03 ; P$ (close fit: RMSEA <.05) $=.99 ;$ FIML $X^{2}(28, N=204)=21.11, P=.82 ; X^{2} / d f$ ratio $\left.=.75\right)$ explaining $82 \%$ of the variance observed in physical activity change. Standardized direct, indirect, and total effect coefficients resulting from the structural analysis are listed in Table 5; standardized direct effects are printed in Figure 3. Among the inactive, weight-challenged aging adults the GTH intervention (ordinally ranked $0=$ control waiting, $1=\mathrm{GTH}$ alone, 2 = GTH-plus supports) led to increases at sevenmonths in self-efficacy $\left(\beta_{\text {[total }]}=.23, P<.001\right)$, in selfregulation $\left(\beta_{\text {[total }]}=.38, P<.001\right)$, and to improved negative outcome expectations $\left(\beta_{\text {[total] }}=-.09, P<.01\right)$. In addition, the GTH had a strong effect on change in participants' physical activity levels at 16 months $\left(\beta_{[\text {total }]}=.58, P<\right.$ .001 ). Participants added more steps and more exercise MET hours/week to their activity routines at higher levels of the intervention. These changes in physical activity and social cognitive variables were independent of participants' age and race $\left(\beta_{\text {[total] }} P<.10\right)$. Gender, on the other hand was an important predictor of change in physical activity $\left(\beta_{\text {[total] }}\right.$ $=.29, P<.05)$; men in the sample made greater increases in physical activity than women.

5.3.2. Social Cognitive Mediators of Effects on Activity Levels. Increases in physical activity at 16 months were predicted by earlier improvements in self-efficacy $\left(\beta_{[\text {total }]}=.41<.05\right)$, which significantly mediated the effect of treatment on physical activity (GTH-SE-PA $a b=.093 ; \mathrm{CI}=.001 ; .22$ ). Changes in self-regulation and positive outcome expectations, however, did not influence change in physical activity $(P>.10)$.

5.3.3. Guide to Health Effects on Weight Management. Although the GTH was not a weight loss intervention, it was hypothesized that participants would not gain typical amounts of weight during the intervention [12]. A final expanded model was evaluated to investigate whether change 


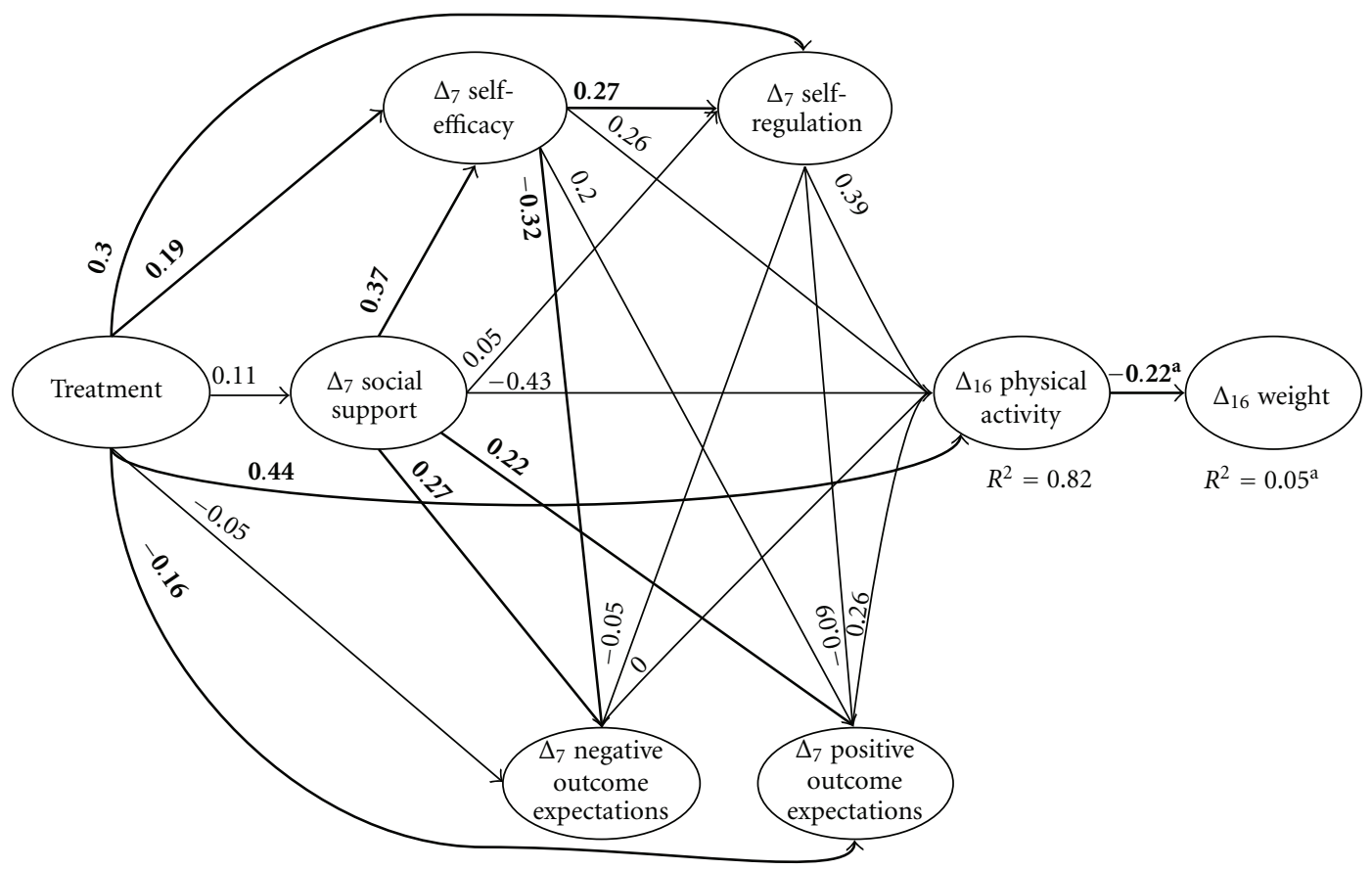

FIGURE 3: Cognitive model of treatment effects among overweight or obese and inactive aging adults $\left(\Delta_{7}=\right.$ change from baseline to 7 months, $\Delta_{16}=$ change from baseline to 16 months; significant effects bolded, ${ }^{a}$ coefficients generated from expanded model of effects on weight).

TABle 4: Descriptive statistics for change in social cognitive variables at 7 months and physical activity and weight change at 16 months.

\begin{tabular}{|c|c|c|c|c|c|c|}
\hline \multirow{3}{*}{ Change variables } & \multicolumn{5}{|c|}{ Treatment condition } & \\
\hline & \multirow[b]{2}{*}{ Control waiting } & & \multirow[b]{2}{*}{ Intervention } & \multirow{2}{*}{\multicolumn{3}{|c|}{$\begin{array}{l}\text { Intervention plus } \\
\text { church supports }\end{array}$}} \\
\hline & & & & & & \\
\hline & M & SD & $\mathrm{M}$ & SD & M & SD \\
\hline \multicolumn{7}{|c|}{ Change at seven-months } \\
\hline Social support & .30 & 1.16 & .14 & 1.04 & .31 & 1.02 \\
\hline SE: barriers & -5.28 & 17.87 & -4.70 & 21.90 & 4.57 & 24.25 \\
\hline SE: daily routine & -8.41 & 18.99 & -6.18 & 18.03 & 2.93 & 17.93 \\
\hline Positive OE & 1.18 & 4.47 & -.11 & 3.98 & -.33 & 5.00 \\
\hline Negative OE & .31 & 3.87 & .37 & 4.46 & -.73 & 3.77 \\
\hline SR: goal setting planning & .28 & .77 & .97 & .95 & .90 & .90 \\
\hline SR: tracking & .47 & 1.26 & 1.67 & 1.21 & 1.50 & 1.44 \\
\hline \multicolumn{7}{|c|}{ Change at 16 months } \\
\hline Steps/day & 427.82 & 2242.03 & 1565.61 & 2964.82 & 2059.03 & 2477.74 \\
\hline Exercise MET hrs/week & 1.02 & 15.35 & -.13 & 13.68 & 10.99 & 22.44 \\
\hline Weight & -.80 & 10.94 & -1.37 & 7.24 & -3.07 & 8.82 \\
\hline
\end{tabular}

SE: self-efficacy, OE: outcome expectations, SR: self-regulation.

in body weight at 16 months was related to treatment, to concomitant changes in physical activity or to the preceding psychosocial changes. In the weight change model, treatment was modeled to influence weight through physical activity and earlier SCT variables (see Figure 3). The weightchange model also provided good fit to the change data: RMSEA $=.00,95 \% \mathrm{CI}=.00 ; .00 ; P$ (close fit: $R M S E A<.05)$ $=1.00 ;$ FIML $X^{2}(40, N=204)=26.29, P=.95 ; X^{2} / d f$ ratio $=.66$ ) explaining $5 \%$ of the observed weight change among overweight or obese, inactive adults in the sample. Higher levels of the GTH intervention led to greater weight loss $\left(\beta_{\text {[total }]}=-.12, P<.05\right)$ at 16 months. Weight change was independent of participants' age, gender and race $(P>.10$, see Table 5).

5.3.4. Social Cognitive Mediators of Effects on Weight Management. Weight loss at 16 months was associated with treatment-related increases in physical activity $\left(\beta_{\text {[total }]}=\right.$ $-.22, P=.01)$ and, albeit marginally, with improvements in self-efficacy at 7 months $\left(\beta_{\text {[total } / \text { indirect }]}=-.09, P<.10\right)$. 
TABLE 5: Social cognitive model of GTH treatment effects among inactive, weight-challenged, aging adults: standardized direct, indirect, and total effects among latent variable.

\begin{tabular}{|c|c|c|c|c|c|c|c|c|c|c|c|}
\hline Latent variable & Effect & Cond. & Age & Race & Gender & SS & SE & NOE & POE & SR & PA \\
\hline Social support (SS) & Direct/total & .11 & -.13 & .12 & $-.22^{* *}$ & & & & & & \\
\hline \multirow{3}{*}{ Self-efficacy (SE) } & Direct & $.19^{* *}$ & .11 & $-.10^{* *}$ & .14 & $.37 * * *$ & & & & & \\
\hline & Indirect & .04 & -.05 & .05 & $-.08^{*}$ & & & & & & \\
\hline & Total & $.23^{* * *}$ & .06 & -.06 & .06 & $.37 * * *$ & & & & & \\
\hline \multirow{3}{*}{ Negative outcome expectations (NOE) } & Direct & -.05 & .14 & .01 & -.02 & $.27^{*}$ & $-.32^{* *}$ & & & & \\
\hline & Indirect & $-.04^{*}$ & -.05 & .05 & -.08 & -.12 & & & & & \\
\hline & Total & $-.09^{* *}$ & .08 & .06 & -.10 & .15 & $-.32 * * *$ & & & & \\
\hline \multirow{3}{*}{ Positive outcome expectations (POE) } & Direct & $-.16^{* *}$ & .09 & $.12^{* *}$ & -.07 & $.22^{*}$ & $.20^{\mathrm{a}}$ & & & & \\
\hline & Indirect & $.07^{*}$ & -.02 & .01 & -.03 & $.07^{*}$ & & & & & \\
\hline & Total & -.09 & .07 & .14 & $-.11^{*}$ & $.29^{* *}$ & $.20^{\mathrm{a}}$ & & & & \\
\hline \multirow{3}{*}{ Self-regulation (SR) } & Direct & $.30^{* * *}$ & -.03 & .04 & -.02 & .06 & $.27^{* *}$ & -.05 & -.09 & & \\
\hline & Indirect & $.08^{*}$ & .00 & -.02 & .02 & .07 & .00 & & & & \\
\hline & Total & $.38^{* * *}$ & -.04 & .01 & .00 & .12 & $.27^{* *}$ & -.05 & -.09 & & \\
\hline \multirow{3}{*}{ Physical activity (PA) } & Direct & $.44^{* * *}$ & -.15 & -.02 & .21 & -.43 & .26 & .00 & $.26^{\mathrm{a}}$ & .39 & \\
\hline & Indirect & .14 & .08 & -.03 & .08 & .22 & $.15^{\mathrm{a}}$ & -.02 & -.04 & & \\
\hline & Total & $.58^{* * *}$ & -.07 & -.05 & $.29^{*}$ & -.21 & $.41^{*}$ & -.02 & .22 & .39 & \\
\hline Body weight & Total & $-.12^{*}$ & .00 & .02 & -.07 & .04 & $-.09^{\mathrm{a}}$ & .02 & -.03 & -.09 & $-.22^{* *}$ \\
\hline
\end{tabular}

${ }^{\mathrm{a}} P<.10 ;{ }^{*} P<.05 ;{ }^{* *} P<.01 ; *^{* *} P<.001$; GTH: Guide to Health; Condi: treatment condition $(0=$ control; $1=\mathrm{GTH}$ only; $2=\mathrm{GTH}$ plus $)$.

Changes in physical activity mediated the effect of the intervention on weight loss (GTH-Physical Activity-Body Weight $a b=-.11 ; \mathrm{CI}=-.25 ;-.02$ ), suggesting inactive, overweight to obese adults over 40 using the GTH lost weight because of increased physical activity which followed from improved psychosocial functioning.

\section{Discussion}

Developing effective interventions to counteract the inactivity and weight gain associated with aging would be enhanced by a broader understanding of how aging may influence psychosocial determinants of physical activity, how theoretical variables may mediate the effect of aging on physical activity and whether interventions targeting important psychosocial variables operate to improve activity levels and weight management as theorized in this population. Among a diverse sample of adults ages 40-92 enrolling in a health promotion study, physical activity declined with age, as did self-efficacy and positive expectations for physical activity. Older participants were less confident in their abilities to overcome barriers to physical activity and to incorporate exercise into their daily routine. On the other hand, with age participants were more likely to experience social support for being physically active, were less likely to anticipate negative outcomes resulting from increased PA, and were more likely to plan, set goals, and to fit exercise into their daily schedules. For weight-challenged, inactive adults over 40, outcomes from a successful SCTbased intervention suggest that increasing self-efficacy is an effective mechanism for increasing physical activity and associated weight management.
The present study incorporated demographic, social cognitive and physical activity variables in a series of latentvariable theoretical models to examine first, the determinants of physical activity in a diverse group of 703 adults ages 40-92 recruited as part of the health promotion GTH study and second, the function of theoretical variables in the SCTbased intervention's effects on physical activity and weight change among the subgroup of over- 40 , weight-challenged inactive participants completing the GTH intervention ( $n$ $=204)$. The sample was racially and socioeconomically diverse and had body composition and activity levels similar to national samples $[30,31]$. Structural modeling analysis indicated the theoretical model specifications provided good fit to the data explaining $18 \%$ of physical activity at baseline, $82 \%$ of physical activity change and $5 \%$ of weight change at 16 months.

Age was the strongest predictor of baseline physical activity-as aging progressed in the sample participants took fewer steps and walked fewer minutes per day. Age also affected important social cognitive variables shown to contribute to physical activity in older populations [1-5]. Consistent with declining activity, self-efficacy for being more active decreased with age. Participants in the 60s+-group reported less confidence in overcoming social, emotional and physical barriers to exercise and in meeting goals of increased exercise than younger participants. Despite declining physical activity and self-efficacy, social support for physical activity increased with age; participants 60 s and above, were more likely to perceive their families as doing the things necessary to remain physically fit than participants in their 40 s or 50s. Similarly, engaging in self-regulatory behaviors increased with age; participants over 60 were more 
likely to plan and keep track of physical activity and more likely to work physical activity into their daily routines than participants in their 40s.

This pattern of age-related effects suggests that even as behavior and self-efficacy decline, supportive environments and increased self-regulation among the aging may present avenues for effective physical activity interventions. Social support has been shown in the current and previous studies with older adults [1] to influence physical activity largely by improving individuals' self-efficacy and use of self-regulatory behaviors indicating effective physical activity treatments should access and enhance aging adults' existing social environments. Similarly, the current findings suggest the selfregulatory behaviors that may be important to physical activity in older adults specifically [1] may become more feasible and acceptable with age. The key issue in efforts to increase physical activity and reduce concomitant health problems among the aging, however, will be to address potential agerelated deterioration of physical activity self-efficacy. Selfefficacy in current and previous studies $[1,2,5]$ has been found to be important to physical activity in older populations; older adults with higher self-efficacy were more active. Thus, interventions counteracting age-related declines in self-efficacy, primarily through enhanced social support, could be expected to help older adults maintain healthier levels of physical activity and avoid related health issues.

Outcomes associated with the GTH intervention (delivered with and without social supports) among inactive and overweight or obese adults over 40 suggest that unlike previous interventions with weaker outcomes for older participants (i.e., $>65$ years, 32 ), the GTH was successful with aging participants regardless of age. Using the GTH program led to greater increases in physical activity and to greater weight loss 16 months after program initiation among weightchallenged, inactive participants over 40. Increased physical activity was a strong predictor of weight loss. Treatment outcomes did not vary by race, but men in the sample made greater changes in physical activity than women even though women made significant changes in social support and positive outcome expectations as a result of the intervention. Physical activity at 16 months was influenced by GTH in part because it led to earlier increases in users' selfefficacy for becoming more active. Although the GTH also increased use of self-regulatory behavior at 7 months among the weight-challenged, inactive, aging adults, these changes did not significantly influence their physical activity levels nine months later. The GTH intervention increased selfefficacy directly rather than through increased social support. Participants' social support was targeted in the intervention through strategies for involving others in their physical activity programs (e.g., find a walking partner, ask family member to remind you of your walking goals) and through churchbased social supports in one study condition, but social support remained unchanged at 7 months. The mastery experiences provided by GTH (increasing physical activity gradually over time, providing positive feedback for change, modifying goals in light of achievements) led directly to users' increased confidence in their abilities to be more active and ultimately to their improved physical activity levels.
Strengths of this study include a verified physical activity measure, a large diverse sample of adults, and the use of SEM. The study has several limitations. First, although large, the sample composition presents two challenges-the high rate of church attendance by participants and the expressed interest in changing health behaviors is not typical of most adults such that the models will need to be verified in a more representative population. Second, the racial and gender differences observed among the participants in psychosocial and physical activity variables suggest differences in how SCT may operate among aging African American and White adults and among aging men and women. The current sample size (162 African American participants at baseline, 62 men at the 16 month followup) could not support the multigroup analyses that could isolate these differences. Finally, the role of outcome expectations as delimited by SCT and defined in the current study was ambiguous as noted in previous research [32]. Although PA, decreased with age, participants were less likely to expect that, PA would interfere with their daily routines or their social obligations. Decreased negative outcome expectations did not, however, enhance PA levels. Granted that SCT suggests outcome expectations might not contribute beyond self-efficacy to behaviors like PA where the behavior is the desired outcome [9], some would suggest that as individuals assess their self-efficacy for PA they necessarily take expected outcomes into account. Older adults who fear injury or who expect to be embarrassed by their reduced physical capacities, for example, will have less confidence in their abilities to be active [33].

Despite these limitations, this study suggests declining physical activity levels in aging adults stem in part from deteriorating self-efficacy for being active despite the supportive social environments and increased self-regulatory vigilance associated with age. The outcomes of the GTH trial suggest that for aging adults suffering from inactivity and weight challenges, SCT-based interventions can effectively increase self-efficacy and self-regulation behavior, leading to increased physical activity and better weight management.

\section{Acknowledgments}

The study conducted at the Center for Research in Health Behavior, Department of Psychology, Virginia Polytechnic Institute and State University was supported by a Grant from the National Cancer Institute (CA79469-02). This project was supported by a Grant from the National Cancer Institute (1R01CA79469-02) to Virginia Polytechnic Institute and State University.

\section{References}

[1] B. J. Ayotte, J. A. Margrett, and J. Hicks-Patrick, "Physical activity in middle-aged and young-old adults: the roles of self-efficacy, barriers, outcome expectancies, self-regulatory behaviors and social support," Journal of Health Psychology, vol. 15, no. 2, pp. 173-185, 2010.

[2] G. F. Dunton, A. A. Atienza, C. M. Castro, and A. C. King, "Using ecological momentary assessment to examine antecedents and correlates of physical activity bouts in adults age 
50+ Years: a pilot study," Annals of Behavioral Medicine, vol. 38, no. 3, pp. 249-255, 2009.

[3] N. Hankonen, P. Absetz, P. Ghisletta, B. Renner, and A. Uutela, "Gender differences in social cognitive determinants of exercise adoption," Psychology and Health, vol. 25, no. 1, pp. 55-69, 2010.

[4] M. Stevens, K. A. P. M. Lemmink, M. J. G. Van Heuvelen, J. De Jong, and P. Rispens, "Groningen Active Living Model (GALM): stimulating physical activity in sedentary older adults; validation of the behavioral change model," Preventive Medicine, vol. 37, no. 6, pp. 561-570, 2003.

[5] E. McAuley, K. S. Morris, R. W. Motl, L. Hu, J. F. Konopack, and S. Elavsky, "Long-term follow-up of physical activity behavior in older adults," Health Psychology, vol. 26, no. 3, pp. 375-380, 2007.

[6] A. Luszczynska, X. Gibbons, and B. F. Piko, "Self-regulatory cognitions, social comparison, and perceived peers' behaviors as predictors of nutrition and physical activity: A comparison among adolescents in Hungary, Poland, Turkey, and USA," Psychology and Health, vol. 19, no. 5, pp. 577-593, 2004.

[7] B. A. Lewis, L. H. Forsyth, B. M. Pinto, B. C. Bock, M. Roberts, and B. H. Marcus, "Psychosocial mediators of physical activity in a randomized controlled intervention trial," Journal of Sport and Exercise Psychology, vol. 28, no. 2, pp. 193-204, 2006.

[8] United States Department of Health and Human Services (USDHHS), Physical Activity and Health: A Report from the Surgeon General, United States Department of Health and Human Services, Centers for Disease Control and Prevention, National Center for Chronic Disease Prevention and Health Promotion, Atlanta, Ga, USA, 1996.

[9] A. Bandura, Self-Efficacy: The Exercise of Control, W H. Freeman, New York, NY, USA, 1997.

[10] A. Bandura, "Health promotion by social cognitive means," Health Education and Behavior, vol. 31, no. 2, pp. 143-164, 2004.

[11] P. M. Barnes and C. A. Schoenborn, "Physical activity among adults: United States, 2000," Advance Data, vol. 333, pp. 1-23, 2003.

[12] R. A. Winett, E. S. Anderson, J. R. Wojcik, S. G. Winett, and T. Bowden, "Guide to health: nutrition and physical activity outcomes of a group-randomized trial of an internet-based intervention in churches," Annals of Behavioral Medicine, vol. 33, no. 3, pp. 251-261, 2007.

[13] J. A. Davis and T. W. Smith, "General social surveys, 19722008 [machine-readable data file]," NORC Edition, National Opinion Research Center, Chicago, Tex, USA, 2009.

[14] American College of Sports Medicine, ACSM's Guidelines for Exercise Testing and Prescription, Williams \& Wilkins, Baltimore, Md, USA, 7th edition, 2005.

[15] J. Myers, W. Herbert, P. Ribisl, and V. F. Froelicher, "A nomogram to predict exercise capacity from a specific activity questionnaire and clinical data," American Journal of Cardiology, vol. 73, no. 8, pp. 591-596, 1994.

[16] C. Tudor-Locke and D. R. Bassett Jr., "How many steps/day are enough? preliminary pedometer indices for public health," Sports Medicine, vol. 34, no. 1, pp. 1-8, 2004.

[17] R. R. Pate, M. Pratt, S. N. Blair et al., "Physical activity and public health: a recommendation from the Centers for Disease Control and Prevention and the American College of Sports Medicine," Journal of the American Medical Association, vol. 273, no. 5, pp. 402-407, 1995.
[18] E. S. Anderson, J. R. Wojcik, R. A. Winett, and D. M. Williams, "Social-cognitive determinants of physical activity: the influence of social support, self-efficacy, outcome expectations, and self-regulation among participants in a church-based health promotion study," Health Psychology, vol. 25, no. 4, pp. 510520, 2006.

[19] B. E. Ainsworth, W. L. Haskell, M. C. Whitt et al., "Compendium of physical activities: an update of activity codes and MET intensities," Medicine and Science in Sports and Exercise, vol. 32, no. 9, supplement, pp. S498-S504, 2000.

[20] K. G. Jöreskog and D. Sorbom, “LISREL 8.8. 2007,” Scientific Software, Chicago, Tex, USA.

[21] R. B. Kline, Principles and Practice of Structural Equation Modeling, Guilford, New York, NY, USA, 2005.

[22] D. M. Murray, S. P. Varnell, and J. L. Blitstein, "Design and analysis of group-randomized trials: a review of recent methodological developments," American Journal of Public Health, vol. 94, no. 3, pp. 423-432, 2004.

[23] R. M. Baron and D. A. Kenny, "The moderator-mediator variable distinction in social psychological research. Conceptual, strategic, and statistical considerations," Journal of Personality and Social Psychology, vol. 51, no. 6, pp. 1173-1182, 1986.

[24] G. N. Holmbeck, "Toward terminological, conceptual, and statistical clarity in the study of mediators and moderators: examples from the child-clinical and pediatric psychology literatures," in Methodological Issues \& Strategies in Clinical Research, A. E. Kazdin, Ed., American Psychological Association, Washington, DC, USA, 3rd edition, 2003.

[25] D. P. MacKinnon, M. S. Fritz, J. Williams, and C. M. Lockwood, "Distribution of the product confidence limits for the indirect effect: program PRODCLIN," Behavior Research Methods, vol. 39, no. 3, pp. 384-389, 2007.

[26] D. P. MacKinnon, C. M. Lockwood, J. M. Hoffman, S. G. West, and V. Sheets, "A comparison of methods to test mediation and other intervening variable effects," Psychological Methods, vol. 7, no. 1, pp. 83-104, 2002.

[27] D. P. MacKinnon, C. M. Lockwood, and J. Williams, "Confidence limits for the indirect effect: distribution of the product and resampling methods," Multivariate Behavioral Research, vol. 39, no. 1, pp. 99-128, 2004.

[28] K. A. Pituch, L. M. Stapleton, and J. Y. Kang, "A comparison of single sample and bootstrap methods to assess mediation in cluster randomized trials," Multivariate Behavioral Research, vol. 41, no. 3, pp. 367-400, 2006.

[29] J. L. Krull and D. P. MacKinnon, "Multilevel mediation modeling in group-based intervention studies," Evaluation Review, vol. 23, no. 4, pp. 418-444, 1999.

[30] K. M. Flegal, M. D. Carroll, C. L. Ogden, and C. L. Johnson, "Prevalence and trends in obesity among US adults, 19992000," Journal of the American Medical Association, vol. 288, no. 14, pp. 1723-1727, 2002.

[31] USDHHS, Healthy People 2010, USDHHS, United States Census Bureau, Washington, DC, USA, 2000, http://www .epodunk.com/ .

[32] D. M. Williams, E. S. Anderson, and R. A. Winett, "A review of the outcome expectancy construct in physical activity research," Annals of Behavioral Medicine, vol. 29, no. 1, pp. 7079, 2005.

[33] D. M. Williams, "Outcome expectancy and self-efficacy: theoretical implications of an unresolved contradiction," Personality and Social Psychology Review, vol. 14, no. 4, pp. 417-425, 2010. 


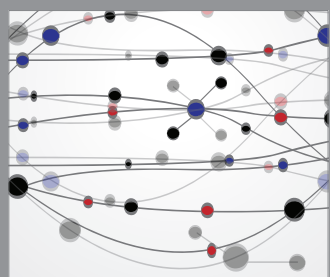

The Scientific World Journal
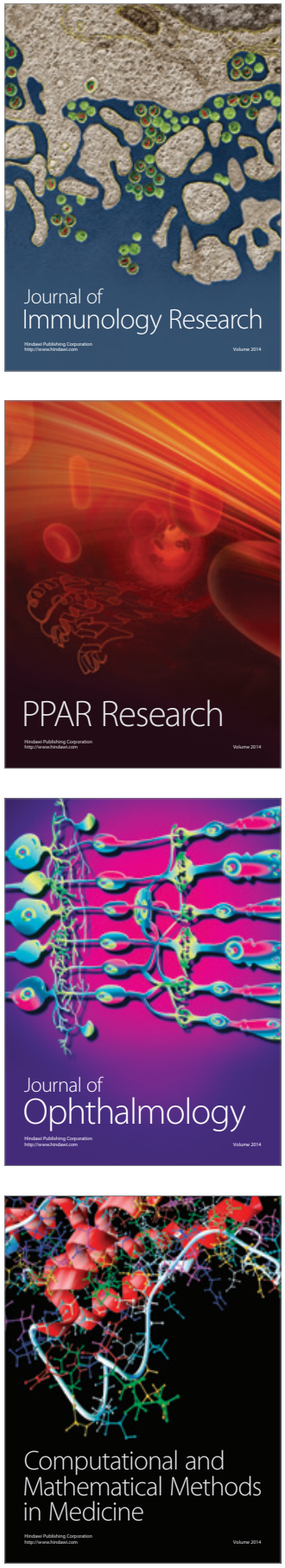

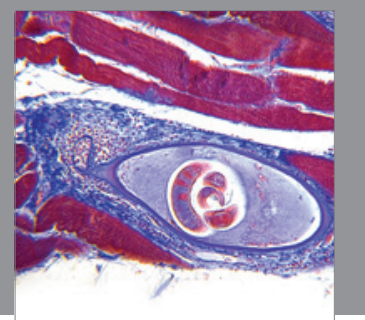

Gastroenterology

Research and Practice
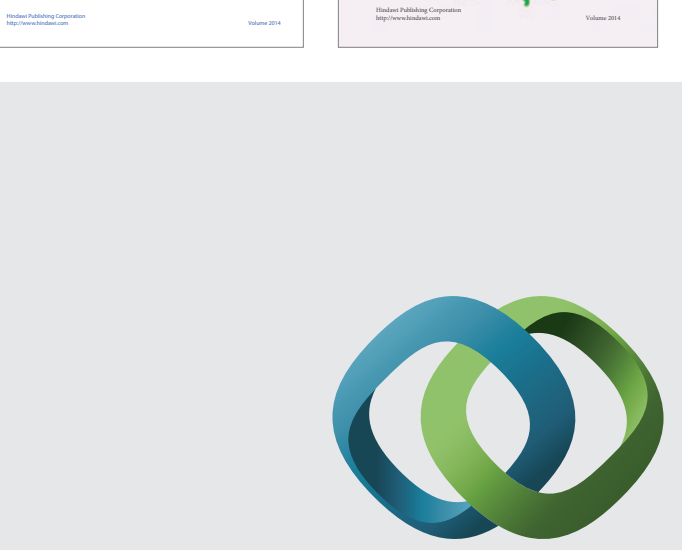

\section{Hindawi}

Submit your manuscripts at

http://www.hindawi.com
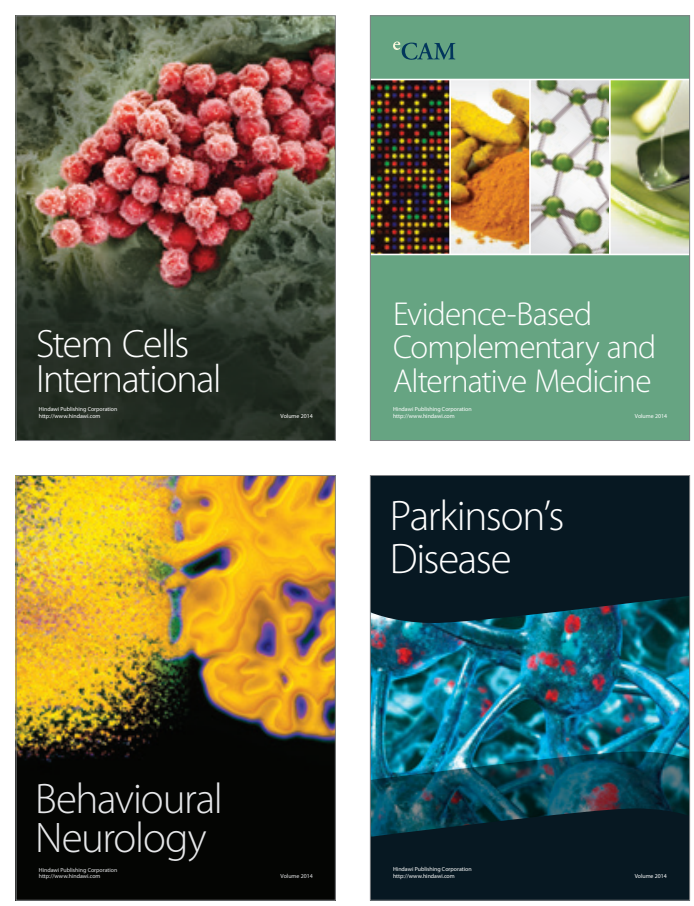

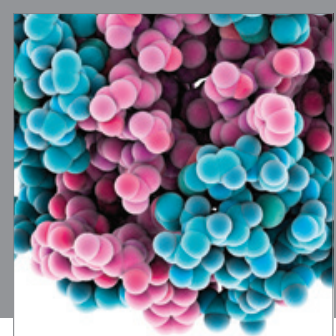

Journal of
Diabetes Research

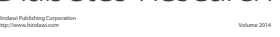

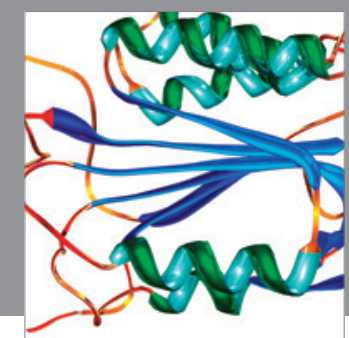

Disease Markers
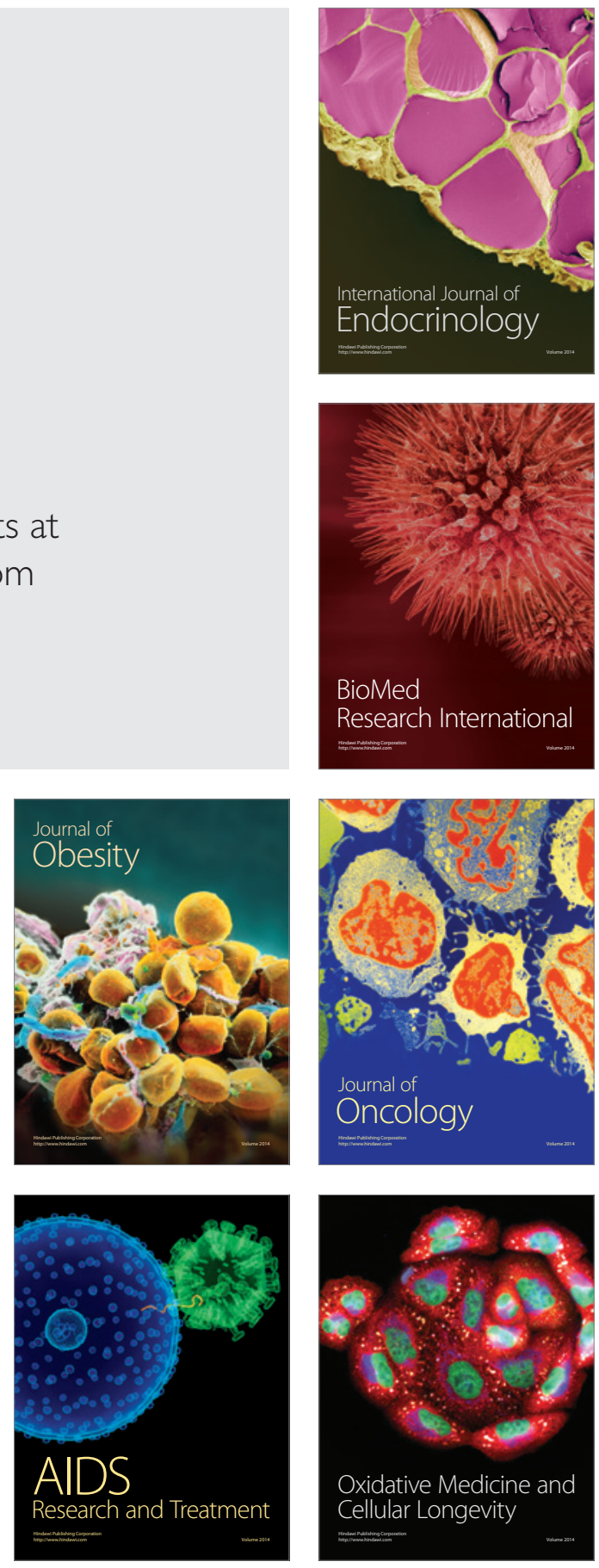